\title{
ChemComm
}

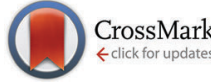

Cite this: Chem. Commun., 2014, 50, 10140

Received 29th May 2014, Accepted 13th July 2014

DOI: $10.1039 / c 4 c c 04130 e$

www.rsc.org/chemcomm

\section{Adsorption of a dihydro-TTF derivative on Au(111) via a thiolate complex bonding to gold adatoms $\dagger$}

\author{
Siddharth J. Jethwa, $\ddagger$ Federico Grillo, Herbert A. Früchtl, Grant J. Simpson, \\ Michael-John Treanor, Renald Schaub, Stephen M. Francis, Neville V. Richardson* \\ and R. Alan Aitken*
}

\begin{abstract}
A dihydro-TTF derivative with four acetyl-protected thiol ligands was synthesised and adsorbed on Au(111) under UHV conditions. Scanning Tunnelling Microscopy (STM) and Infrared (IR) spectroscopy show that self-organised structures are formed following annealing to $333 \mathrm{~K}$, with each pair of bidentate thiolate ligands bonding to a single gold adatom in a $\mathrm{S}-\mathrm{Au}_{\mathrm{ad}}-\mathrm{S}$ complex. Due to the lack of a direct orbital overlap between the dihydro-TTF moieties and the surface, relatively little charge transfer between TAT-TTF and the gold surface occurs.
\end{abstract}

Tetrathiafulvalene (TTF $)^{1}$ has been the subject of extensive research in the field of molecular electronics ${ }^{2}$ because of its ability to act as an excellent electron donor. TTF is able to undergo two reversible one-electron oxidation processes, with a low ionisation potential because of the aromatic stability of its cationic and dicationic states. ${ }^{3}$ A key consideration in molecular electronics is the interface between the electroactive molecule and the electrode. ${ }^{4}$ Accordingly, TTF $^{5}$ and its derivatives ${ }^{6}$ have been studied in detail on surfaces. However, for many applications, direct interaction of the electroactive moiety with the electrode is undesirable, since this leads to a broadening of the molecular orbitals. $^{7}$ The ubiquitous sulfur-gold covalent bond has been used to provide a more convenient through-bond electrical contact. ${ }^{8}$ A number of studies have shown that a thiolate bond to a metal surface can be formed through cleavage of the acetylthio functionality at the sulfur-acetyl bond in solution ${ }^{9}$ or upon surface annealing. ${ }^{10}$ Considering the large interest in TTF derivatives, to date only a limited number of surface studies have been conducted on functionalised TTF molecules capable of forming thiolate bonds with gold surfaces. ${ }^{11}$ In particular, a detailed STM study is currently lacking. In the light of research showing that gold adatoms are involved in

School of Chemistry, University of St Andrews, Fife, Scotland, KY16 9ST, UK.

E-mail:neville.richardson@st-andrews.ac.uk, raa@st-andrews.ac.uk

$\dagger$ Electronic supplementary information (ESI) available: Synthetic and characterisation details, STM data of acetyl radicals, and DFT calculation details. See DOI: 10.1039/c4cc04130e

\# Present address: Interdisciplinary Nanoscience Center (iNano) and Department of Physics and Astronomy, Aarhus University, 8000 Aarhus C, Denmark.

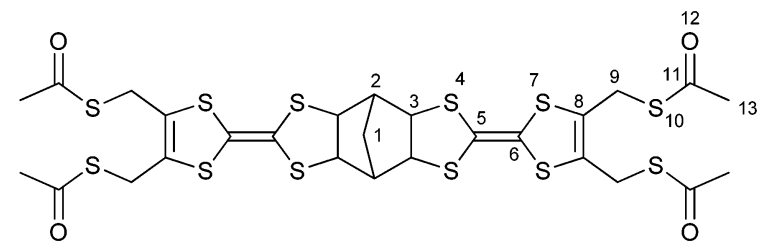

Fig. 1 Chemical structure of TAT-TTF; atom numbering is to assist for IR assignment.

thiolate bonding complexes, ${ }^{12}$ a comprehensive understanding of the binding geometry of thiolate functionalised TTF derivatives on $\mathrm{Au}(111)$ is required.

The tetra-acetylthio dihydro-TTF derivative (TAT-TTF) shown in Fig. 1 was synthesised (described in ESI 1-3†). The molecule consists of two dihydro-TTF rings separated by a norbornane bridge. The $-\mathrm{CH}_{2}-$ spacer group in the ligand allows a degree of flexibility for binding to the substrate. ${ }^{13}$ The TTF-skeleton is formed using the synthetic procedure outlined by Aitken et al., ${ }^{14}$ with the terminal groups converted into the acetylthio functionality using the method defined by Hudhomme et al. ${ }^{15}$ for related TTF-derivatives. It is important to note that the two TTF groups are partially saturated at the norbornane ring; however partially saturated TTF groups are still able to act as electron donors. ${ }^{16}$ Due to the lack of aromatisation gain upon oxidation of the 1,3-dithiolane ring, dihydro-TTF molecules are correspondingly weaker electron donors. The presence of the acetylthio groups is indicated in the transmission IR spectrum of TAT-TTF (Fig. 2a), by the strong carbonyl stretching band at $1687 \mathrm{~cm}^{-1}$. Prior to use, the TAT-TTF sample was degassed at $341 \mathrm{~K}$ for 24 hours. Under UHV conditions, an $\mathrm{Au}(111)$ crystal held at $298 \mathrm{~K}$ was exposed to TAT-TTF evaporated from a molecular doser heated to $420 \mathrm{~K}$ (Fig. 2b); experimental conditions are given in ESI 4. $\dagger$ In line with previous studies,${ }^{17}$ the absence of a strong carbonyl stretching mode at $1687 \mathrm{~cm}^{-1}$ indicates that the sulfur-acetyl bond in TATTTF has been cleaved. However, an extra peak appears in the Reflection-Absorption Infrared (RAIR) spectrum at $2908 \mathrm{~cm}^{-1}$ that is assigned to the $\mathrm{CH}_{3}$ stretching mode of an acetyl radical species. ${ }^{18}$ The $\mathrm{C}=\mathrm{O}$ bond of the acetyl radical is believed to lie 
(a)

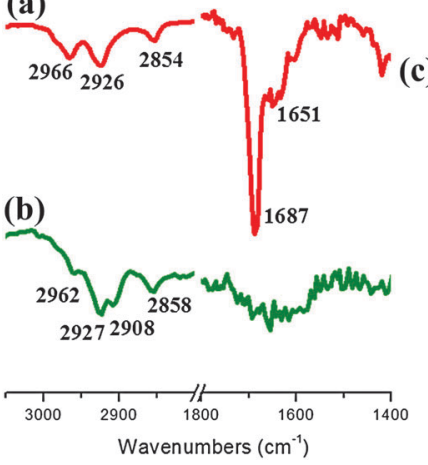

Fig. 2 (a) Transmission IR spectrum of TAT-TTF in KBr; (b) RAIR spectrum of $\mathrm{Au}(111)$ surface after TAT-TTF sublimation at $341 \mathrm{~K}$ and (c) Vibrational assignments for TAT-TTF. Atom numbering as in Fig. 1.

parallel to the surface and hence infrared inactive according to the surface selection rule. This assignment is supported by STM data (see ESI $5 \dagger$ ), where spherical features $4.0 \AA$ A in diameter are observed that compare well to the dimensions of acetyl groups adsorbed on $\mathrm{Ag}(100),{ }^{10}$ following surface-mediated cleavage of the acetylthio functionality of porphyrin molecules. From the current set of data it is not clear whether cleavage of the sulfuracetyl bond is surface-mediated and occurs upon adsorption, or is a result of the thermal decomposition of TAT-TTF during the sublimation process. The continued presence of a $\mathrm{CH}_{2}$ antisymmetric stretching mode of the norbornane cage $\left(2962 \mathrm{~cm}^{-1}\right)$, and the $\mathrm{CH}_{2}$ stretching modes at the $\mathrm{C}_{9}$ position $\left(2927 \mathrm{~cm}^{-1}\right.$ and $2858 \mathrm{~cm}^{-1}$ ), lead to the conclusion that the central functional moiety of the TAT-TTF molecule remains intact upon adsorption at $298 \mathrm{~K}$.

STM images of the surface were acquired at $77 \mathrm{~K}$. Following deposition at $298 \mathrm{~K}$, only disordered areas of organic material are observed. However, annealing to $333 \mathrm{~K}$ for 1 hour results in ordered 'ribbon' type structures becoming visible (Fig. 3a). In the case of $\mathrm{Au}(111)$, it is reported that adatom-thiolate complexes are highly mobile at room temperature and can restructure the gold surface. ${ }^{19}$ Therefore the annealing process is likely to facilitate the diffusion of these adatom complexes. This gives them sufficient mobility to coalesce through weak hydrogen bonding/ van der Waals interactions, and allows the subsequent formation of the observed ribbon structures. Further annealing to $373 \mathrm{~K}$ results in the disappearance of the ribbon structures, with only molecular 'debris' remaining. The ribbon structures are found exclusively within the $f c c$ regions within the gold herringbone reconstruction, as observed for other thiol species, ${ }^{20}$ and consist of a repeated motif composed of four circular features; the two terminal features are brighter than the two central ones. These features are aligned along the [ $52-7]$ direction of the substrate as indicated in Fig. 3b. Submolecular bright features in studies of thioether ${ }^{21}$ and thiophenyl ${ }^{22}$ derivatives on $\mathrm{Au}(111)$ have been interpreted as gold adatoms being incorporated into surface structures. The brighter features are measured as $1.6 \AA$ higher than the surface plane and $0.2 \AA$ higher than the two central features. The height is significantly shorter than that measured for other systems $(2.6 \AA)^{21,22}$ where a gold adatom is thought
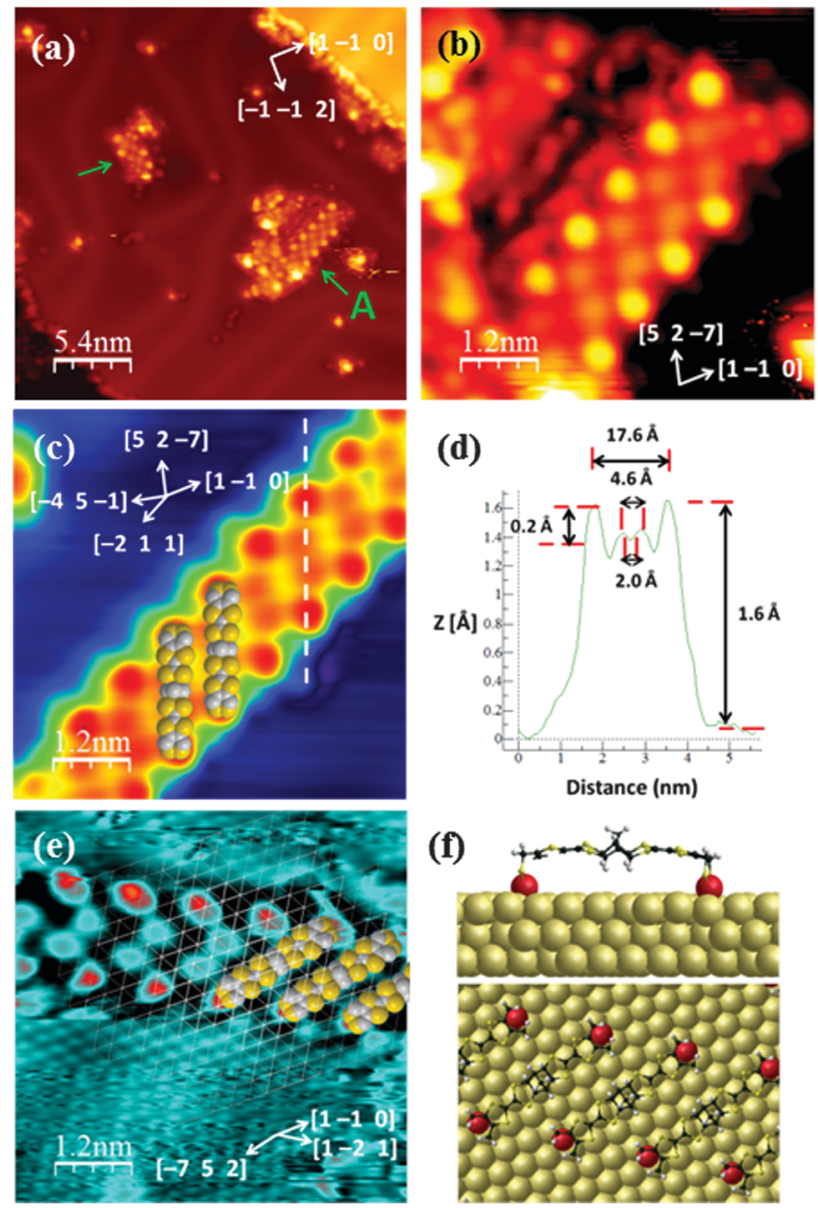

Fig. 3 STM images of TAT-TTF islands on $\mathrm{Au}(111)$ after annealing to $333 \mathrm{~K}$ (a) $-0.6 \mathrm{~V}, 0.06 \mathrm{nA}, 27 \times 27 \mathrm{~nm}^{2}$; (b) Island A, $-0.6 \mathrm{~V}, 0.06 \mathrm{nA}, 6 \times 6 \mathrm{~nm}^{2}$; (c) $-0.9 \mathrm{~V}, 0.08 \mathrm{nA}, 6 \times 6 \mathrm{~nm}^{2}$; (d) Line profile taken along white line indicated in (c); (e) $-0.2 \mathrm{~V}, 0.13 \mathrm{nA}, 6 \times 6 \mathrm{~nm}^{2}$; (f) Optimised DFT geometry of TAT-TTF bound via gold adatoms (red) to surface atoms (dark yellow).

to be present. However this can be rationalised, since the measured height reflects contributions from the electronic structure and the physical 'topography' of the combined molecule/surface. Since the RAIR data indicate cleavage of the sulfur-acetyl bond, then it is possible that a direct thiolate bond is formed from the bidentate sulfur ligands at either end of TAT-TTF to a single gold adatom, as determined by previous experimental observations of dimeric $\mathrm{S}-\mathrm{Au}_{\mathrm{ad}}-\mathrm{S}$ binding complexes. ${ }^{12}$ Accordingly, a scaled space-filling model of TAT-TTF, that is cleaved at the sulfur-acetyl bond, has been overlaid upon an enhanced contrast STM image of a molecular ribbon (Fig. 3c). Here the sulfur ligands are overlaid over the bright terminal features, where it is considered that a single gold adatom is located. The two central features correspond to the electron density located on the dihydro-TTF rings. Note that the molecules must be aligned along the $\left[\begin{array}{lll}5 & 2 & -7\end{array}\right]$ direction, and not along the $[-45-1]$ direction, in order to agree with the termination of the chain in Fig. 3b. The ribbons themselves are aligned along the $\left\langle\begin{array}{lll}1 & 1 & 2\end{array}\right\rangle$ directions of the underlying gold lattice. An important point to note from Fig. $3 \mathrm{c}$ is the apparent skew in electron density of the two central features towards neighbouring brighter features. This, we speculate, 
is the result of weak $\mathrm{C}-\mathrm{H} \cdots \mathrm{S}$ hydrogen bonding interactions between the sulfur atoms in the TTF-rings and the $-\mathrm{CH}_{2}-\left(\mathrm{S}_{4}\right.$ on the TTF-ring, $\mathrm{C}_{9}$ on the $-\mathrm{CH}_{2}-$ spacer) and $\mathrm{CH}\left(\mathrm{S}_{7}\right.$ on the TTF-ring, $\mathrm{C}_{2}$ on the norbornane cage) groups of adjacent molecules. Additional $\mathrm{S} \cdots \mathrm{S}$ van der Waals interactions between TTF-rings are also likely to assist in the formation of the TAT-TTF ribbon structures. Such interactions are recognised to play a key role in determining the crystal packing of molecules containing the TTF moiety. ${ }^{23}$ The lateral spacing of molecules, between 2.5-3.0 $\AA$ (see ESI $5 \dagger)$, is compatible with the range over which $\mathrm{C}-\mathrm{H} \cdots \mathrm{S}(\leq 3.15 \AA)$ and $\mathrm{S} \cdots \mathrm{S}(\leq 4.0 \AA)$ interactions can occur. ${ }^{23}$ The line-profile shown in Fig. 3d shows that the peak-to-peak separation of $17.6 \AA$ corresponds to the length of TAT-TTF minus the acetyl groups of 17.3 A, extrapolated from a related molecule (see ESI $3 \dagger$ ). Fig. $3 \mathrm{e}$ shows a remarkable image of a TAT-TTF ribbon, where both the molecule and the underlying gold surface atoms are clearly resolved. Here the molecules are clearly aligned along the [-7 5 2 2$]$ direction. However, even though the overlaid (111) lattice suggests the gold adatom is located in a three-fold hollow site, the exact binding site of the sulfur-gold adatom complex cannot be derived from the image alone.

In order to find the optimal binding configuration of TATTTF, periodic DFT calculations were performed using the VASP software package ${ }^{24}$ (see ESI $6 \dagger$ for details). The optimised geometry is shown in Fig. 3f. Here, TAT-TTF is cleaved at the sulfur-acetyl bond as indicated by the RAIR results, with the two sulfur atoms of the bidentate ligands on either end of the molecule bound to a single gold adatom in an $\mathrm{S}-\mathrm{Au}_{\mathrm{ad}}-\mathrm{S}$ complex. This is in agreement with previous models of thiolate-gold adatom complexes. ${ }^{12}$ The molecule is orientated along the [-7 5 2 2 ] direction, with its short-axis tilted by around $15^{\circ}$ to the surface plane. The two calculated $\mathrm{S}-\mathrm{Au}_{\mathrm{ad}}$ bond lengths are measured as $2.33 \AA$ and $2.40 \AA$ A. These compare well with the DFT calculated $\mathrm{S}-\mathrm{Au}_{\mathrm{ad}}$ bond lengths of methylthiolate-gold adatom complexes of $2.30-2.50 \AA^{12}$ The gold adatoms are located in three-fold hollow sites, whilst the terminal sulfur atoms are located approximately above two-fold bridge sites. The latter does not agree with the DFT calculations of Häkkinen et al. ${ }^{12}$ where the sulfur atoms are located on atop sites. This may be due to the extra rigidity of the thiolate ring structure in TAT-TTF. It is clear from the calculations that the sulfur atoms of the dihydro-TTF rings are considered to be too distant $(\geq 5.18 \AA)$ to interact directly with the surface. Fernandez-Torrente et al. ${ }^{5}$ have shown that direct overlap of the electronic orbitals of the sulfur atoms of TTF with the gold surface results in a partial charge transfer from the molecule to the surface, causing Coulombic repulsion between neighbouring molecules. Quite clearly this is not the case for the current system. As noted above, dihydro-TTF derivatives are weaker electron donors than fully unsaturated systems. Nevertheless, it is likely that charge-transfer processes may occur through the thiolate-gold adatom complex rather than directly between the dihydro-TTF moiety and the surface. The Bader charges analysis ${ }^{25}$ of the model allows us to rationalise this point (see ESI $6 \dagger$ ). The TAT-TTF molecule adsorbed via adatoms on the $\mathrm{Au}(111)$ surface has a total charge of -0.38 , with the terminal sulfurs being the most negative atoms. This is compared with the charge of the molecule within the gas phase Au(TAT-TTF)Au complex that has a total charge of -0.33 , mainly transferred from the two $\mathrm{Au}$ adatoms. Therefore the charges on the dihydro-TTF moiety are nearly unperturbed and the small change has to be ascribed to the superposition of the thiolategold adatom-surface orbitals.

In conclusion, an acetylthio functionalised dihydro-TTF derivative, TAT-TTF, was synthesised and successfully adsorbed on a $\mathrm{Au}(111)$ surface. Ordered molecular ribbons are formed upon annealing to $333 \mathrm{~K}$. Evidence of $\mathrm{C}-\mathrm{H} \cdots \mathrm{S}$ and $\mathrm{S} \cdots \mathrm{S}$ intermolecular interactions between neighbouring molecules to form such structures has been demonstrated. Relatively little charge transfer between TAT-TTF and the gold surface occurs, due to the lack of a direct orbital overlap between the dihydro-TTF moieties and the surface. The well-defined binding conformation of the bidentate $-\mathrm{CH}_{2} \mathrm{~S}$ - termini to a single gold adatom provides an effective tunnelling barrier between the TAT-TTF molecule and the $\mathrm{Au}(111)$ surface. This could be of interest in future molecular electronic applications which rely on quantum tunnelling, such as molecular rectifiers and resonant tunnelling diodes, ${ }^{26}$ where the isolation of the electroactive moiety from the surface is considered of utmost importance.

The authors wish to thank the SPIRIT grant for funding of this project. EaStCHEM is thanked for computational support via the EaStCHEM Research Computing Facility.

\section{Notes and references}

1 N. Martín, Chem. Commun., 2013, 49, 7025 and references therein.

2 R. Andreu, J. Garin, J. Orduna, R. Alcala and B. Villacampa, Org. Lett., 2003, 5, 3143; D. Canevat, M. Salle, G. Zhang, D. Zhang and D. Zhu, Chem. Commun., 2009, 2245; M. Mas-Torrent and C. Rovira, Chem. Rev., 2011, 111, 4833.

3 F. Wüdl, G. M. Smith and E. J. Hufnagel, J. Chem. Soc. D, 1970, 1453.

4 S. Braun, W. R. Salaneck and M. Fahlman, Adv. Mater., 2009, 21, 1450; S. Karthäuser, J. Phys.: Condens. Matter, 2011, 23, 013001; G. Witte and Ch. Wöll, Phys. Status Solidi A, 2008, 205, 497.

5 I. Fernandez-Torrente, S. Monturet, K. J. Franke, J. Fraxedas, N. Lorente and J. I. Pascual, Phys. Rev. Lett., 2007, 99, 176103; Y. Wang, C. Urban, J. R. Fernandez, J. M. Gallego, R. Otero, N. Martin, R. Miranda, M. Alcami and F. Martin, J. Phys. Chem. A, 2011, 115, 13080.

6 M. Abdel-Mottaleb, E. Gomar-Nadal, M. Surin, H. Uji-i, W. Mamdouh, J. Veciana, V. Lemaur, C. Rovira, J. Cornil, R. Lazzaroni, D. B. Amalbino, S. De. Feyter and F. C. De Schryver, J. Mater. Chem., 2005, 15, 4601; C. Urban, D. Ecija, Y. Wang, M. Trelka, I. Preda, A. Vollmer, N. Lorente, A. Arnau, M. Alcami, L. Soriano, N. Martin, F. Martin, R. Otero, J. M. Gallego and R. Miranda, J. Phys. Chem. C, 2010, 114, 6503.

7 P. E. Kornilovitch, A. M. Bratkovsky and R. S. Williams, Phys. Rev. B: Condens. Matter Mater. Phys., 2002, 66, 165436.

8 S. N. Yaliraki, M. Kemp and M. A. Ratner, J. Am. Chem. Soc., 1999, 121, 3428; J. Ulrich, D. Esrail, W. Pontius, L. Venkataraman, D. Millar and L. H. Doerrer, J. Phys. Chem. B, 2006, 110, 2462.

9 Y. Kang, D. J. Won, S. R. Kim, K. Seo, H. S. Choi, G. Lee, Z. Noh, T. S. Lee and C. Lee, Mater. Sci. Eng., C, 2004, 24, 43.

10 O. P. Vaughan, M. Turner, F. J. Williams, A. Hille, J. K. Sanders and R. M. Lambert, J. Am. Chem. Soc., 2006, 128, 9578.

11 C. M. Yip and M. D. Ward, Langmuir, 1994, 10, 549; R. Yuge, A. Miyazaki, T. Enoki, K. Tamada, F. Nakamura and M. Hara, J. Phys. Chem. B, 2002, 106, 6894; E. Gomar-Nadal, G. K. Ramachandran, F. Chen, T. Burgin, C. Rovira, D. B Amalbino and S. M. Lindsay, J. Phys. Chem. B, 2004, 108, 7213; Y. Yokota, K. Fukui, T. Enoki and M. Hara, J. Am. Chem. Soc., 2007, 129, 6571; L. R. Ditzler, C. Karunatilaka, V. R. Donaru, H. Y. Liu and A. V. Tivanski, J. Phys. Chem. C, 2010, 114, 4429.

12 O. Voznyy, J. J. Dubowski, J. T. Yates and P. Maksymovych, J. Am. Chem. Soc., 2009, 131, 12989; P. Maksymovych and J. T. Yates, J. Am. Chem. Soc., 2008, 130, 7518; H. Grönbeck, H. Häkkinen and R. L. Whetten, J. Phys. Chem. C, 2008, 112, 15940. 
13 N. Garg and T. R. Lee, Langmuir, 1998, 14, 3815.

14 R. A. Aitken, L. Hill and P. Lightfoot, Tetrahedron Lett., 1997, 38, 7927. 15 P. Hudhomme, S. Le Moustarder, C. Durand, N. G. Planas, N. Mercier, P. Blanchard, E. Levillain, M. Allain, A. Gorgues and A. Riou, Chem. - Eur. J., 2001, 7, 5070.

16 H. Nishikawa, T. Morimoto, T. Kodama, I. Ikemoto, K. Kikuchi, J. Yamada, H. Yoshino and K. Murata, J. Am. Chem. Soc., 2002, 124, 730 .

17 P. Piotowski, J. Pawlowska, B. Palys, S. Sek, R. Bilewicz and A. Kaim, J. Electrochem. Soc., 2013, 160, H28.

18 M. R. Nimloss, J. A. Soderquist and G. B. Ellison, J. Am. Chem. Soc., 1989, 111, 7675.

19 M. Yu, N. Bovet, C. J. Satterley, S. Bengio, K. R. J. Lovelock, P. K. Milligan, R. G. Jones, D. P. Woodruff and V. Dhanak, Phys. Rev. Lett., 2006, 97, 166102; S. J. Stranick, A. N. Parikh, D. L. Allara and
P. S. Weiss, J. Phys. Chem., 1994, 98, 11136; M. J. Esplandiu, M. L. Carot, F. P. Cometto, V. A. Macagno and E. M. Patrito, Surf. Sci., 2006, 600, 155.

20 Y. Liu and V. Ozolins, J. Phys. Chem. C, 2012, 116, 4738.

21 Y. Pan, B. Yang, C. Hulot, S. Blechert, N. Nilius and H. J. Freund, Phys. Chem. Chem. Phys., 2012, 14, 10987.

22 B. Yang, Y. Pan, X. Lin, N. Nilius, H. J. Freund, C. Hulot, A. Giraud, S. Blechert, S. Tosoni and J. Sauer, J. Am. Chem. Soc., 2012, 116, 24558.

23 J. J. Novoa, M. C. Rovira, C. Rovira, J. Veciana and J. Tarrés, Adv. Mater., 1995, 7, 233.

24 G. Kresse and J. Furthmüller, Phys. Rev. B: Condens. Matter Mater. Phys., 1996, 54, 11169.

25 W. Tang, E. Sanville and G. Henkelman, J. Phys.: Condens. Matter, 2009, 21, 084204.

26 J. C. Ellenbogen and J. C. Love, Proc. IEEE, 2000, 83, 386. 